\title{
Comparison of radial basis functions in evaluating the Asian option
}

\author{
F. Zhai, K. Shen \& E. Kita \\ Graduate School of Information Sciences, Nagoya University, Japan
}

\begin{abstract}
Some researchers have presented the application of radial basis function approximation to the evaluation of option contracts.

In a previous study, the authors described the evaluation of Asian options by using radial basis function approximation. The numerical results indicated that the computational accuracy depended on the radial basis function and the reciprocal multi-quadric function was better than the multi-quadric one.

So, in this study, some radial basis functions are applied to the evaluation of the Asian option of one asset. We compare the multi-quadric, the reciprocal multiquadric, and Gaussian functions. The results show that the reciprocal multiquadric function and Gaussian function give better numerical results and the reciprocal multi-quadric function is better than the others.
\end{abstract}

\section{Introduction}

Recently, the financial derivatives are dealt widely and the importance is expanded. The importance of the derivative transaction is increasing for the adequate sharing of the financial risk. The option transaction is one of the most important financial derivatives and therefore, several schemes have been presented by many researchers for their pricing $[1,2]$.

Several financial options have been developed; European option, American option, Look-Back option, Exotic option and so on. In the previous study [3], the authors described the formulation for evaluation of the Asian option. The results showed that the computational accuracy of the present algorithm depended on the radial basis function to be employed for the evaluation. In this study, three kinds of the radial basis functions are applied for evaluation of Asian option and then, the computational accuracy and cost are compared. 
The remaining of the paper is organized as follows. The algorithm for the valuation of the Asian option is described in section 2. The numerical examples are shown in section 4. Finally, the obtained results are summarized in section 5.

\section{Formulation}

\subsection{Governing equation and strike condition}

First, we will define the time-average value of the asset price $S$ as the function:

$$
I=\int_{0}^{t} S(\tau) d \tau
$$

In the European-type average strike option, the payoff depends on the difference between the time-average value and the asset price on the expiration date. The governing differential equation of the option is given as:

$$
\frac{\partial V}{\partial t}+S \frac{\partial V}{\partial I}+\frac{1}{2} \sigma^{2} S^{2} \frac{\partial^{2} V}{\partial S^{2}}+r S \frac{\partial V}{\partial S}-r V=0
$$

If the function $R$ is defined from the asset price $S$ as

$$
\begin{aligned}
R & =\frac{1}{S} \int_{0}^{t} S(\tau) d \tau \\
& =\frac{I}{S}
\end{aligned}
$$

the price $V$ is given as

$$
V(S, R, t)=S H(R, t) .
$$

Substituting equations (3) and (4) to equation (2), we have

$$
\frac{\partial H}{\partial t}+F H=0
$$

where the operator $F$ is defined as

$$
F=\frac{1}{2} \sigma^{2} R^{2} \frac{\partial^{2}}{\partial R^{2}}+(1-r) R \frac{\partial}{\partial R} .
$$

The payoff condition of the average strike option on the expiration date $t=T$ is defined as follows, in the case of European-call type,

$$
\max \left(S-\frac{1}{T} \int_{0}^{t} S(\tau) d \tau, 0\right)
$$

and, in the case of European-put type,

$$
\max \left(\frac{1}{T} \int_{0}^{t} S(\tau) d \tau-S, 0\right) .
$$

where $\max \left(a_{1}, a_{2}\right)$ means the bigger one among $a_{1}$ and $a_{2}$. 
Now, we consider the pricing of the average strike option in the call-type.

Substituting equations (3) and (4) to (7), we have the payoff condition on the expiration date $t=T$;

$$
S H(R, T)=S \max \left(1-\frac{R}{T}, 0\right),
$$

and therefore,

$$
H(R, T)=\max \left(1-\frac{R}{T}, 0\right) .
$$

Finally, the governing equation and the boundary condition of the average strike option are given by equation (5) and (9), respectively.

\subsection{Solution using RBF}

Discretizing the equation (5) with the Crank-Nicolson Scheme, we have

$$
\frac{H(t+\Delta t)-H(t)}{\Delta t}+(1-\theta) F H(t+\Delta t)+\theta F H(t)=0
$$

where the parameter $\theta$ is taken in the range of $0 \leq \theta \leq 1$.

Defining the parameters $H(t)=H^{m}$ and $H(t+\Delta t)=H^{m+1}$, we have

$$
A H^{m+1}=B H^{m}
$$

where

$$
\begin{aligned}
& A=1+(1-\theta) \Delta t F \\
& B=1-\theta \Delta t F
\end{aligned}
$$

The price $H$ governed with the equation (5) is approximated with the RBF function as

$$
H=\sum_{n=1}^{N} \lambda_{n} \phi_{n}
$$

where $N$ and $\lambda_{j}$ denote the total number of data points and the unknown parameters, respectively.

Substituting equation (12) to equation (11), we have

$$
\begin{aligned}
A \sum_{n=1}^{N} \lambda_{n}^{m+1} \phi_{n} & =B \sum_{n=1}^{N} \lambda_{n}^{m} \phi_{n} \\
\sum_{n=1}^{N} A \phi_{n} \lambda_{n}^{m+1} & =\sum_{n=1}^{N} B \phi_{n} \lambda_{n}^{m}
\end{aligned}
$$


136 Boundary Elements and Other Mesh Reduction Methods XXIX

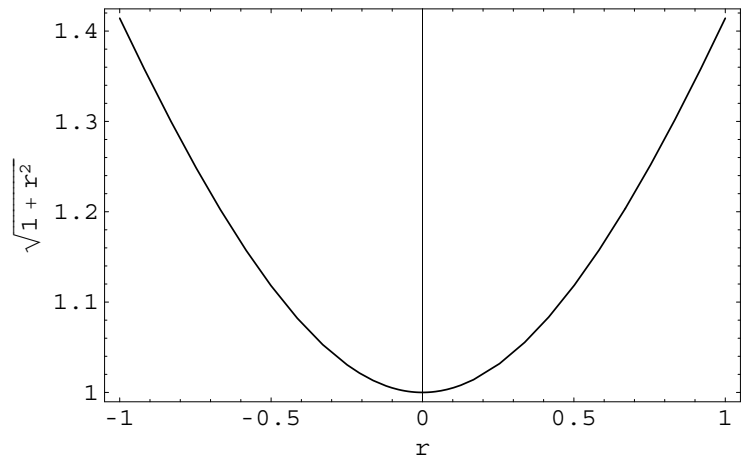

Figure 1: Multi-quadric RBF.

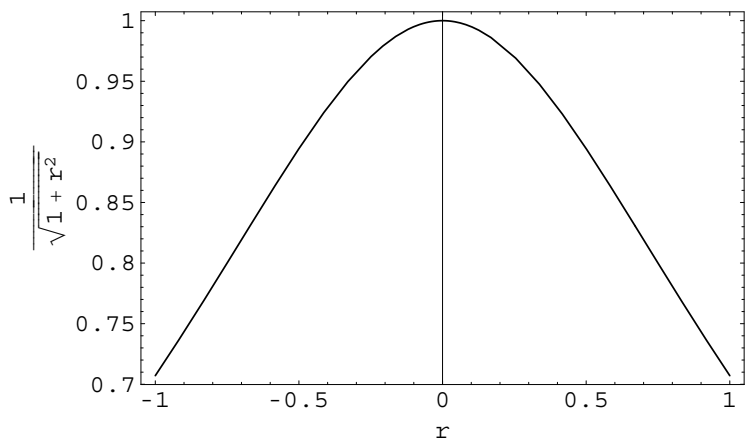

Figure 2: Reciprocal multi-quadric RBF.

\section{Radial basis functions}

In this study, the following radial basis functions are employed for the valuation of the options.

Multi-quadric RBF: The function is defined as follows. The distribution of $c=1$ is shown in Fig. 1.

$$
\phi\left(R, R_{j}\right)=\sqrt{c^{2}+\left\|R-R_{j}\right\|^{2}}
$$

Reciprocal multi-quadric RBF: The function is defined as follows. The distribution of $c=1$ is shown in Fig. 2 .

$$
\phi\left(R, R_{j}\right)=\frac{1}{\sqrt{c^{2}+\left\|R-R_{j}\right\|^{2}}}
$$




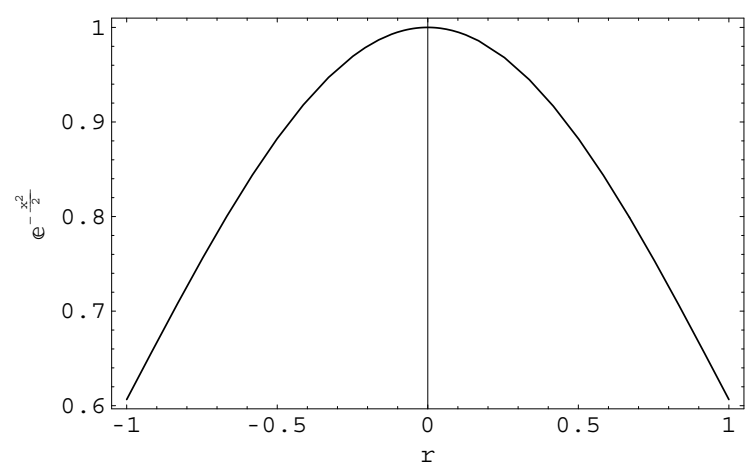

Figure 3: Gaussian RBF.

Gaussian RBF: The function is defined as follows. The distribution of $c=1$ is shown in Fig. 3.

$$
\phi\left(R, R_{j}\right)=\exp \left(-\frac{\left\|R-R_{j}\right\|^{2}}{2 c^{2}}\right)
$$

\subsection{Algorithm}

The algorithm of the solution procedure is defined as

1. Distribute $N$ data points on $0 \leq R \leq R_{\max }$ and discretize $0 \leq t \leq T$ with $T / M$.

2. Solve equation (12) to evaluate $H^{T}$ on the expiration date $t=T$.

3. Approximate $H^{T}$ by equation (12) to evaluate $\lambda_{n}^{T}$ on the expiration date $t=T$.

4. $t \leftarrow T-\Delta t$.

5. Solve equation (13) to estimate $\lambda_{n}^{t}$.

6. $t \leftarrow t-\Delta t$.

7. IF $t \neq 0$, go to step 5 .

8. Evaluate $H^{0}$ from equation (12) and $\lambda_{n}^{0}$ on the date $t=0$.

\section{Numerical example}

The simulation parameters are defined as shown in Table 1 . The total number of the data points are 101. They are distributed uniformly in the range of $0 \leq R \leq 1.0$. For comparison with the finite difference solutions, the time-step size is taken as $\Delta t=0.0005$; the number of the time-step is $M=1000$. The computations are performed with a personal computer of Intel Core Solo $1.06 \mathrm{GHz}$ and $1 \mathrm{~Gb}$-main memory and Mathematica version 5.2.

First, CPU times are compared in Table 2. The RMQ-RBF computation is fastest among them and followed with, in turn, MQ-RBF, FDM and Gaussian. Especially, the CPU times of MQ and RMQ are smaller than the FDM. 
Table 1: Parameters for numerical result.

\begin{tabular}{ll}
\hline Expiration date & $T=0.5$ [year] \\
Risk free interest rate & $r=0.1$ \\
Volatility & $\sigma=0.4$ \\
Crank-Nicholson $\theta$-weighted method & $\theta=0.5$ \\
Maximum R & $R_{\max }=1.0$ \\
Number of timestep(timestep size) & $M=1000(\Delta t=0.0005)$ \\
Number of stock data points & $N=101$ \\
\hline
\end{tabular}

Table 2: CPU Time.

\begin{tabular}{cc}
\hline Function & CPU Time (s) \\
\hline Multi-quadric & 13.453 \\
Reciprocal Multi-quadric & 11.046 \\
Gaussian & 1158.26 \\
\hline FDM & 14.593 \\
\hline
\end{tabular}

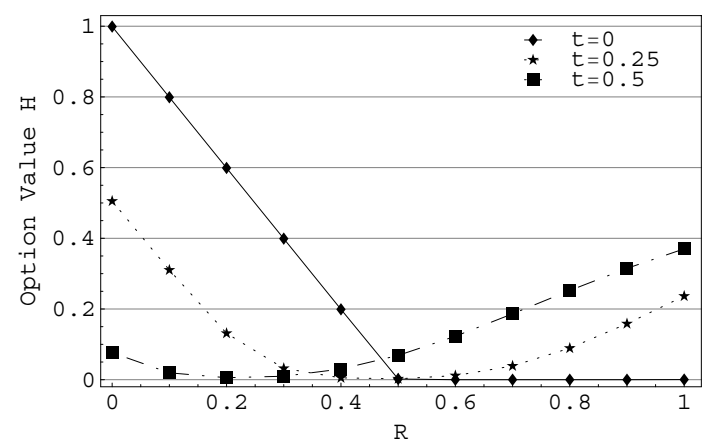

Figure 4: Multiquadrics RBF, $c=0.04$.

Next, the computational accuracy is compared. The parameter $c$ of the MQRBF (14) is taken as $c=0.04$, which was determined from the condition number of the coefficient matrix [3]. Figure 4 shows the option value $H$ at $t=0 \mathrm{C} t=\frac{T}{2}=0.25 \mathrm{C} t=T=0.5$. The abscissa and the ordinate denote $R$ and $H$, respectively. The results by finite difference method are shown in Fig. 5. We notice that the MQ-RBF solution error increases for larger value of $R$. 


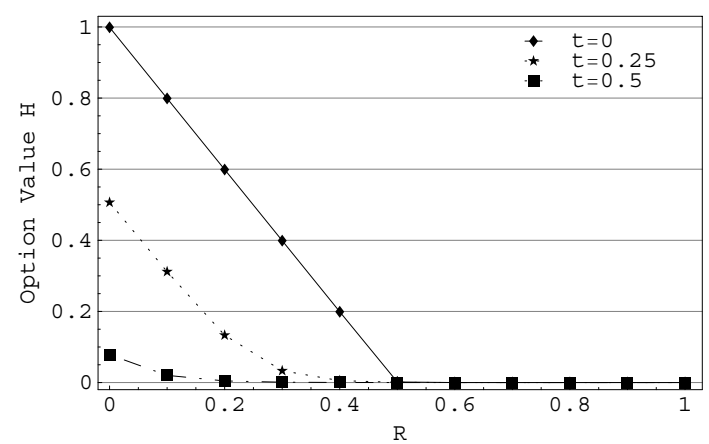

Figure 5: FDM.

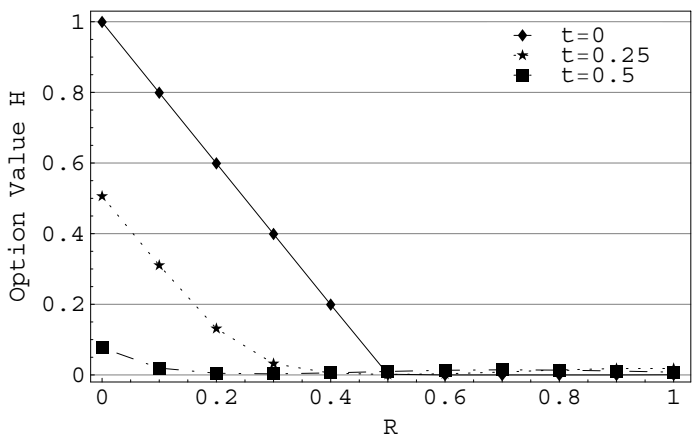

Figure 6: Reciprocal Multiquadric RBF, $c=0.04$.

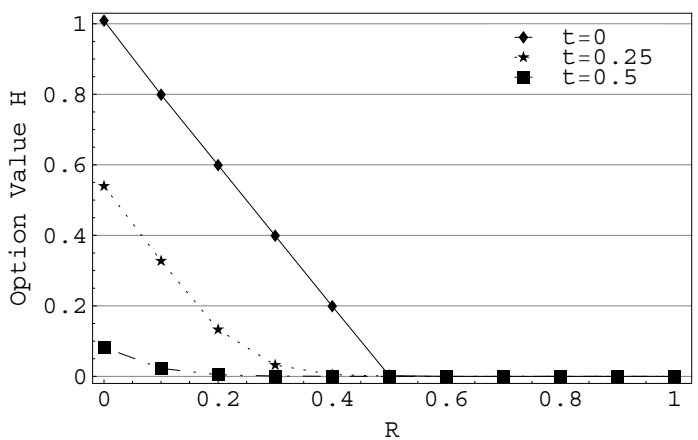

Figure 7: Gaussian RBF, $c=0.01$.

Next, the results by RMQ-RBF are discussed. The RMQ-RBF solutions are shown in Fig. 6. When comparing 6 with Fig. 4, we notice that the RMQ-RBF solution is improved for larger value of $R$. 
The results by Gaussian RBF are shown in Fig. 7. In this case, the parameter $c$ is changed to $c=0.01$ in order to improve the accuracy.

\section{Conclusions}

This paper described the comparison of the RBFs for evaluation of the Asian option. The formulation for evaluating the option was described and the numerical results were shown. Multi-quadric (MQ), reciprocal multi-quadric (RMQ), and Gaussian RBFs were compared in numerical examples. From the viewpoint of the CPU time, the RMQ-RBF computation was faster than the others and followed with, in turn, MQ-RBF, FDM and Gaussian. Especially, the CPU times of MQ and RMQ are smaller than the FDM. When the RBF solutions is compared with FDM one, the solutions by RMQ and Gaussian RBFs agree well with the FDM one. However, the MQ solution has relatively large error. Finally, the authors can conclude that the RMQ-RBF is more adequate for valuation of Asian option from the both sides of the CPU time and the computational accuracy.

\section{References}

[1] G. Courtadon. A more accurate finite difference approximation for valuation of options. Journal of Financial and Quantitative Analysis, Vol. 17, pp. 697$703,1982$.

[2] P. Wilmott, J. Dewynne, and S. Howison. Option Pricing: Mathematical Models and Computation. Oxford Financial Press, 1993.

[3] E. Kita, Y. Goto, F. Zhai, and K. Shen. Evaluation of Asian option by using rbf approximation. In C. A. Brebbia and J. T. Katsikadelis, editors, Boundary Elements XXVIII (Skiathos, Greece, 2006), pp. 33-40, 2006. 